\title{
Heart Modeling, Computational Physiology and the IUPS Physiome Project
}

\author{
Peter J. Hunter \\ Auckland Bioengineering Institute (ABI), University of Auckland, New Zealand \\ p.hunter@auckland.ac.nz
}

\begin{abstract}
The Physiome Project of the International Union of Physiological Sciences (IUPS) is attempting to provide a comprehensive framework for modelling the human body using computational methods which can incorporate the biochemistry, biophysics and anatomy of cells, tissues and organs. A major goal of the project is to use computational modelling to analyse integrative biological function in terms of underlying structure and molecular mechanisms. To support that goal the project is developing XML markup languages (CellML \& FieldML) for encoding models, and software tools for creating, visualizing and executing these models. It is also establishing web-accessible physiological databases dealing with model-related data at the cell, tissue, organ and organ system levels. Two major developments in current medicine are, on the one hand, the much publicised genomics (and soon proteomics) revolution and, on the other, the revolution in medical imaging in which the physiological function of the human body can be studied with a plethora of imaging devices such as MRI, CT, PET, ultrasound, electrical mapping, etc. The challenge for the Physiome Project is to link these two developments for an individual - to use complementary genomic and medical imaging data, together with computational modelling tailored to the anatomy, physiology and genetics of that individual, for patient-specific diagnosis and treatment.
\end{abstract}

\section{References}

[1] Hunter, P.J., Borg, T.K.: Integration from proteins to organs: The Physiome Project. Nature Reviews Molecular and Cell Biology 4, 237-243 (2003)

[2] Crampin, E.J., Halstead, M., Hunter, P.J., Nielsen, P.M.F., Noble, D., Smith, N.P., Tawhai, M.: Computational physiology and the Physiome Project. Exp. Physiol. 89, $1-26$ (2004)

[3] Hunter, P.J., Nielsen, P.M.F.: A strategy for integrative computational physiology. Physiology 20, 316-325 (2005)

[4] Hunter, P.J.: Modeling living systems: the IUPS/EMBS Physiome Project. Proceedings of the IEEE 94, 678-691 (2006)

[5] http://www.cellml.org 